\title{
A NOVEL DENOISING ALGORITHM FOR AIRBORNE LIDAR POINT CLOUD BASED ON EMPIRICAL MODE DECOMPOSITION
}

\author{
Z. Hui ${ }^{1}$, P. Cheng ${ }^{1 *}$, L. Wang ${ }^{1}$, Y. Xia ${ }^{1}, \mathrm{H} . \mathrm{Hu}^{1}, \mathrm{X}^{1} \mathrm{Li}^{1}$ \\ ${ }^{1}$ Faculty of Geomatics, East China University of Technology, Nanchang, China - huizhenyang2008@163.com, \\ pgcheng1964@163.com, wleyang@163.com,20432xyp@163.com,15179582932@163.com,2434288475@qq.com
}

Commission III, WG III/5

KEY WORDS: Airborne LiDAR point cloud, Outliers, Denoising, Empirical mode decomposition

\begin{abstract}
:
Denoising is a key pre-processing step for many airborne LiDAR point cloud applications. However, the previous algorithms have a number of problems, which affect the quality of point cloud post-processing, such as DTM generation. In this paper, a novel automated denoising algorithm is proposed based on empirical mode decomposition to remove outliers from airborne LiDAR point cloud. Comparing with traditional point cloud denoising algorithms, the proposed method can detect outliers from a signal processing perspective. Firstly, airborne LiDAR point clouds are decomposed into a series of intrinsic mode functions with the help of morphological operations, which would significantly decrease the computational complexity. By applying OTSU algorithm to these intrinsic mode functions, noise-dominant components can be detected and filtered. Finally, outliers are detected automatically by comparing observed elevations and reconstructed elevations. Three datasets located at three different cities in China were used to verify the validity and robustness of the proposed method. The experimental results demonstrate that the proposed method removes both high and low outliers effectively with various terrain features while preserving useful ground details.
\end{abstract}

\section{INTRODUCTION}

Airborne LiDAR (Light Detection and Ranging) has become an important remote sensing means for capturing the threedimensional geometry of the Earth (Lin and Zhang, 2014). By integrating the global positioning system, the inertial navigation system and the laser scanning sensor, huge amount of point clouds reflected from Earth's surface can be obtained (Yang et al., 2016). These point clouds consist of three parts, namely ground points, object points and noisy points (Meng et al., 2010). Both ground and object points can be used for lots of applications, such as digital elevation model (DTM) generations, road extractions, forest investigations, etc., while noisy points are undesired measurements.

The noisy points can be categorized into high and low outliers. The high outliers are always elevated points that are generally generated by birds and low flying aircraft. The low outliers normally originate from multi-path and errors in the laser range finder and thus do not belong to the landscape (Sithole and Vosselman, 2003). The existence of noisy points will always bring about some negative effects, including that (1) the quality of DTM generation may be affected by the noisy points, especially the low outliers, since most of the filtering algorithms always assume that the lowest points in the local areas must belong to ground; (2) the rendering of point cloud based on elevation will be influenced due to the maximal or minimal elevations of outliers; and (3) mass of noisy point will incur low three-dimensional model reconstruction quality and decrease the degree of automation.

To avoid the influences mentioned above, lots of researchers have made contributions on airborne LiDAR point cloud denoising. These denoising algorithms can be classified into three categories, namely algorithms based on morphological operations (Chen et al., 2007; Mongus and Zalik, 2012; Mongus et al., 2014; Li et al., 2013; Li et al., 2014), elevation thresholds setting (Haugerud and Harding, 2001; SilvánCárdenas and Wang, 2006; Hui et al., 2016) and interpolation fitting (Brovelli et al., 2002; Wang et al., 2009), respectively.

Although lots of denoising algorithms have been put forward in recent years, most of them are only byproducts of realizing other applications, such as filtering, modeling, feature extracting among others. Moreover, there are some unresolved problems in the above-mentioned denoising algorithms. The main problem of the algorithms based on morphological operations is how to choose appropriate structuring elements for denoising. The performance of threshold-setting-based approaches relies heavily on the threshold, which can only be determined by trial and error. The methods based on interpolation fitting always mislabel some ground points as noise points in the abrupt terrains, which affect the quality of point cloud post-processing, such as DTM generation.

To overcome these problems, a novel denoising algorithm based on empirical mode decomposition (EMD) has been proposed in this paper. EMD owns many good characteristics, including completeness, self-adaptability, data-driven, model-free, etc. Thus, EMD can be applied to nonlinear and nonstationary data sets such as airborne LiDAR point clouds. In this paper, point clouds are decomposed into a series of intrinsic mode functions (IMFs) and one residual. By using OTSU algorithm (Otsu, 1979) the noise-dominated IMFs were determined, and then these IMFs were then filtered according to soft thresholding method. After reconstructing using all the processed IMFs and residual, noise points were detected automatically. The main contributions of this paper are as follows

\footnotetext{
${ }^{*}$ Corresponding author
} 
(1) Propose a new theoretical background denoising algorithm for point clouds on the basis of EMD; (2) Integrate morphological operations with EMD to realize envelope estimation, which will decrease the computational complexity; and (3) Apply OTSU algorithm to IMFs to extract noisedominated components automatically.

The remainder of this paper was organized as follows. Section 2 elaborated the principle of the proposed method, while experiments and analysis were undertaken in Section 3. At last, conclusions were drawn at the end of this paper

\section{METHODOLOGY}

Figure 1 depicts the flow chart of the proposed method. Airborne LiDAR point cloud was first transformed into digital surface model (DSM) using nearest neighbor interpolation, since raster data own the strength of high efficiency and are easy to implement. And then the DSM was decomposed into a series of IMFs and one residual. To filter the noise-dominated IMFs, this paper innovatively adopted OTSU algorithm to determine high-frequency modes (noise-dominated components) automatically. Finally, a comparison of the elevation differences between reconstructed elevations and observed elevations was conducted to detect noise points effectively.

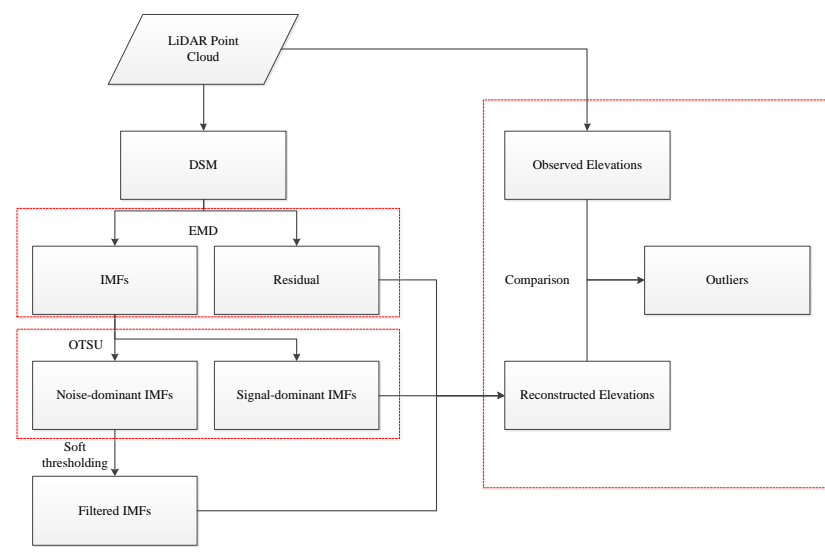

Figure 1. Flow chart of the proposed method

\subsection{IMFs Extraction by Sifting Process}

The EMD was first introduced by Huang et al. (1998) for analyzing data from nonstationary and nonlinear processes, and now has been successfully applied to hyperspectral image classification in remote sensing areas (Demir and Erturk, 2010; Demir et al., 2011; Erturk et al., 2013; He et al., 2014). The EMD involves the decomposition of the two-dimensional (2-D) signal $S(x, y)$ into a series of IMFs by a sifting process, as shown in Figure 2. The sifting process is conducted in an iterative manner. The process of EMD is repeated until the number of extrema of residual is less than 2 or its iterations reach the pre-defined maximum times. The results of EMD are composed of several IMFs and one residual term given by

$$
S(x, y)=\sum_{i=1}^{n} I M F_{i}+r_{n}
$$

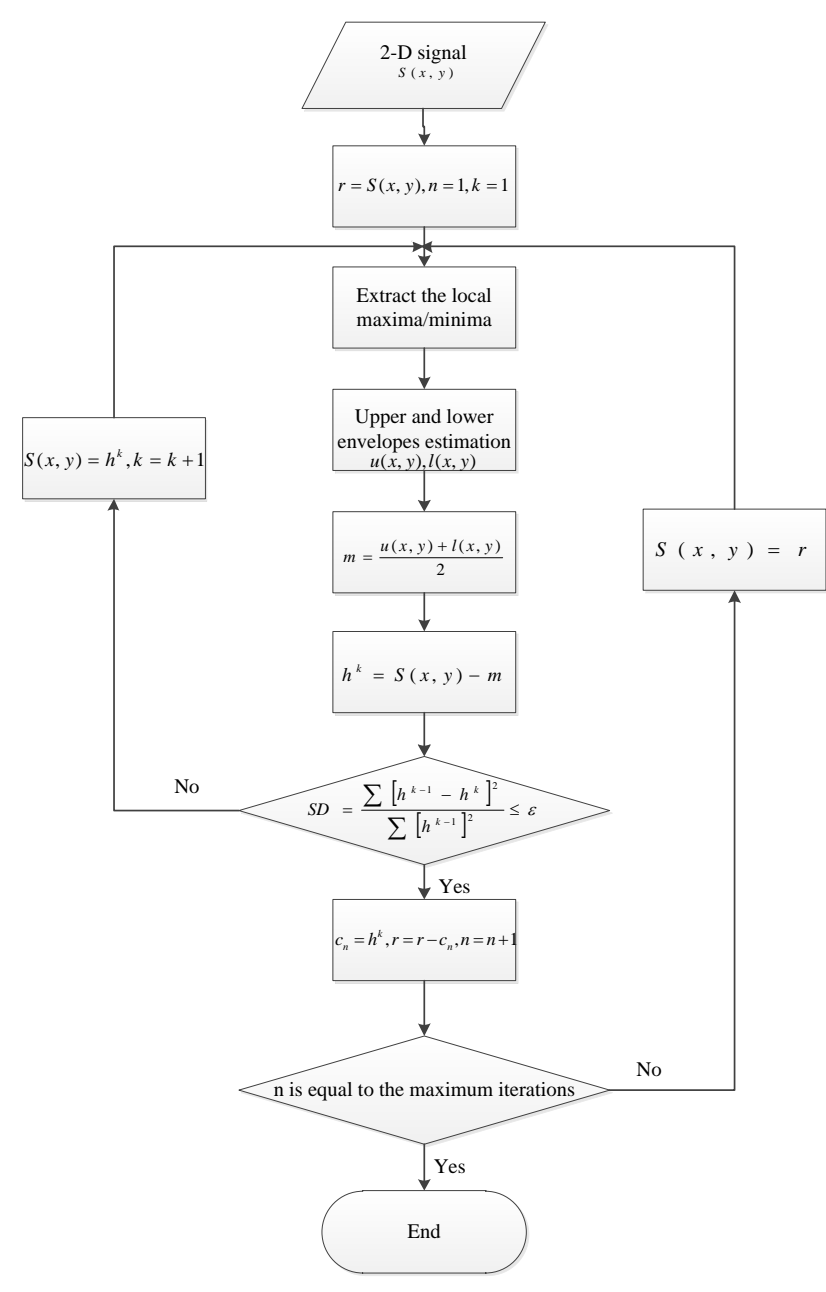

Figure 2. Flow chart of sifting process

\subsection{Envelopes Estimation Using Morphological Operations}

Among the sifting process, one important procedure is to obtain the upper and lower envelopes of the data set. The envelope estimation procedure is the main part affecting the EMD implementation efficiency. The traditional algorithms always use cubic spline to estimate the upper and lower envelopes, which is efficient for one dimensional signal. However, this paper applied the EMD to huge number of point clouds. Therefore, if cubic spline is still applied for envelope estimating, the sifting process will be time consuming. Hence, this paper adopted morphological operations to obtain upper and lower envelopes due to their high implementation efficiency and low computation complexity.

The morphological operations used in this paper involve morphological dilation and morphological erosion. As we know, the upper envelopes are obtained by interpolating local maxima, while the lower envelopes are achieved by interpolating local minima. To make it feasible for point clouds, morphological dilation was adopted instead of interpolating maxima given by

$$
u(x, y)=\delta_{w}(S)=\max _{(x, y) \in w}(S(x, y))
$$

where

$$
\begin{aligned}
& w=3 \times 3 \\
& \delta_{w} \text { denotes the morphological dilation }
\end{aligned}
$$


In this way, all the local maxima can be accepted as components of upper envelope. In terms of lower envelope, it can be obtained using morphological erosion given by

$$
l(x, y)=\gamma_{w}(S)=\min _{(x, y) \in w}(S(x, y))
$$

where $\quad w=3 \times 3$

$\gamma_{w}$ denotes the morphological erosion

\subsection{High-frequency Modes Based on OTSU Algorithm}

The result of EMD consists of a series of IMFs. These IMFs can be classified as two categories, namely high-frequency modes corresponding to noise-dominated components and lowfrequency modes corresponding to signal-dominated components. Thus, there will be a mode indexed by $k$ separating these two kinds of modes given by

$$
S(x, y)=\sum_{i=1}^{k} I M F_{i}+\sum_{i=k+1}^{n} I M F_{i}+r_{n}
$$

This paper applied the OTSU algorithm to the mode index determination. The OTSU algorithm was first presented by Otsu (1979) for image classification by selecting optimal threshold automatically. The OTSU algorithm first dichotomizes the pixels into two classes, namely background and objects by a threshold at level $k$. The optimal threshold can be obtained by maximizing the variance between background and objects classes. This paper adopted a similar principle to determine the high-frequency modes. Firstly, the first IMFs ( $\left.I M F_{i}, i=1,2, \cdots, k\right)$ and the residual term compose the class of 'background', whereas the last IMFs $\left(I M F_{i}, i=k+1, k+2, \cdots, n\right)$ and the residual term compose the class of 'objects'.

According to the principle of OTSU, when the between-class variance reaches maximum, the optimal threshold will be achieved given by

$$
\begin{aligned}
& k=\underset{1 \leq k \leq n}{\arg \max }\left(\sigma^{2}(k)\right) \\
& \sigma^{2}(k)=\omega_{1}\left(\mu_{1}-\mu\right)^{2}+\omega_{2}\left(\mu_{2}-\mu\right)^{2}
\end{aligned}
$$

where

$$
\begin{aligned}
& \omega_{1}=k / n \\
& \mu_{1}=\left(\sum_{i=1}^{k} I M F_{i}+r_{n}\right) / N \\
& \omega_{2}=(n-k) / n \\
& \mu_{2}=\left(\sum_{i=k+1}^{n} I M F_{i}+r_{n}\right) / N \\
& N \text { is the size of the signal }
\end{aligned}
$$

\subsection{4 Filtering of high-frequency modes}

These high-frequency modes need to be filtered so as to make the recovered signal free from noise. The filtering methods always rely on wavelet thresholding including soft thresholding and hard thresholding, which are defined as Equations (6)-(7): Soft thresholding:

$$
\hat{W}_{j, k}= \begin{cases}\operatorname{sign}\left(W_{j, k}\right)\left(\left|W_{j, k}\right|-t h\right) & \left|W_{j, k}\right| \geq t h \\ 0 & \left|W_{j, k}\right|<t h\end{cases}
$$

Hard thresholding:

$$
\hat{W}_{j, k}= \begin{cases}W_{j, k} & \left|W_{j, k}\right| \geq t h \\ 0 & \left|W_{j, k}\right|<t h\end{cases}
$$

where $\quad W_{j, k}$ is wavelet coefficient

$\operatorname{sign}(\cdot)$ is a sign function

th is the threshold at $j$ level

th is generally defined as Equation (8)

$$
t h=\sigma_{j} \sqrt{2 \ln N}
$$

where $\sigma_{j}$ is the standard deviation at $j$ level

$N$ is the length of the signal

This paper adopted soft thresholding method to filtering these noise-dominated modes. Thus, a variant of soft thresholding for EMD is defined as Equation (9)

$$
I M F_{i}^{\prime}= \begin{cases}\operatorname{sigh}\left(I M F_{i}\right)\left(\left|I M F_{i}\right|-t h\right) & \left|I M F_{i}\right| \geq t h \\ 0 & \left|I M F_{i}\right|<t h\end{cases}
$$

where $I M F^{\prime}$ is the IMF after thresholding

\subsection{Criterion for Noise Points Detection}

These high-frequency modes need to be filtered so as to make the recovered signal free from noise. This paper adopted soft thresholding method to filtering these noise-dominated modes. The 2D signal can be reconstructed using the filtered highfrequency modes and low-frequency modes. The recovered $2 \mathrm{D}$ signal can be seen as noise-free signal. Based on mapping relationships between the 2D signal (DSM) and point clouds, the recovered elevation of each point can be obtained. Generally speaking, the noise points own two characteristics, namely abrupt elevation and scatter. Thus, the noise points can be detected based on Equation (6)

$$
\text { Noise }=\left\{\begin{array}{l}
p \mid \operatorname{abs}\left(z(p)-z^{\prime}(p)\right)>\operatorname{th} 1 \& \& \\
\operatorname{Num}\left(\operatorname{abs}\left(z(p)-z\left(p_{i}\right)\right)<t h 2\right)<t h 3
\end{array}\right\}
$$

where

$$
\begin{aligned}
& z(p) \text { is the observed elevation } \\
& z^{\prime}(p) \text { is recovered elevation } \\
& \text { th1 is the elevation difference threshold } \\
& \text { th2 is threshold for detecting neighboring points } \\
& \text { th3 is the number threshold }
\end{aligned}
$$

\section{EXPERIMENTAL RESULTS AND ANALYSIS}

Three different datasets used in practice were tested to evaluate the performance of the proposed denoising method under varying terrain features. The first dataset (sample1) covers diverse land-use and land-cover types including residential buildings, roads, forests and farmlands as shown in Fig. 3 (a). The second dataset (sample2) characterizes by modern architecture with low and high-storey buildings as shown in Fig. 
4 (a). The third dataset (sample3) is dominated by mountains and forests. Among them some rivers and small lakes existed as shown in Fig. 5 (a).

To show the performance of the proposed method, three horizontal profiles of the digital surface model (DSM) before denoising were selected from the three datasets as shown in Fig.3 (b), Fig.4 (b) and Fig.5 (b). From these three onedimensional profiles we can find that both high and low outliers own abrupt elevations comparing with their neighboring points. Moreover, these outliers are generally scattered. After applying the proposed method to these three datasets, the corresponding denoising results were achieved as shown in Fig.3 (c), Fig.4 (c) and Fig.5 (c). The three corresponding horizontal profiles elaborate that the proposed method eliminated both high and low outliers effectively while preserving useful ground details.

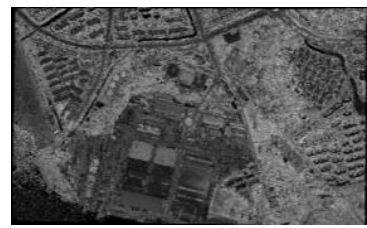

(a)

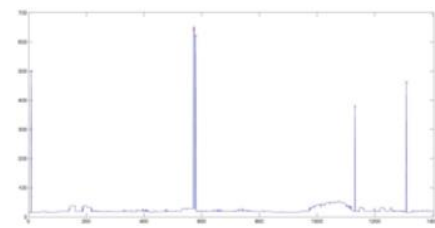

(b) (c)

Figure 3. Denoising and result of the first dataset: (a) Gray-scale image rendered using intensity values; (b) A horizontal profile of the DSM (red crossed symbols indicate outliers); and (c) The corresponding result after denoising.

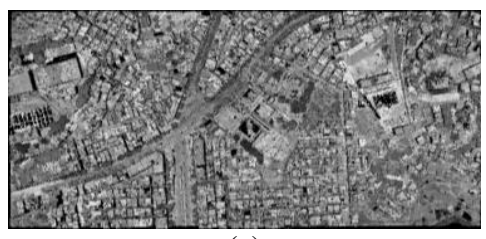

(a)

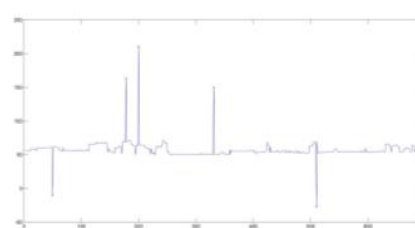

(b) (c)

Figure 4. Denoising and result of the first dataset: (a) Gray-scale image rendered using intensity values; (b) A horizontal profile of the DSM (red crossed symbols indicate outliers); and (c) The corresponding result after denoising.

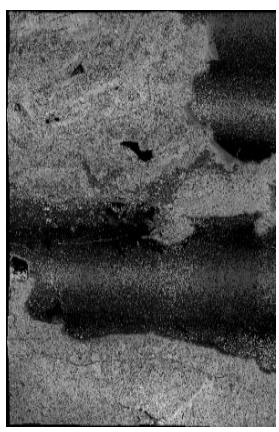

(a)

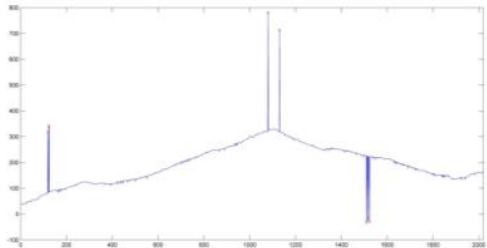

(b)

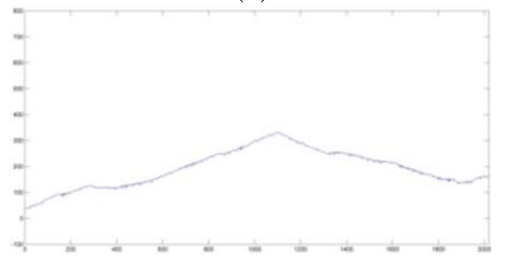

(c)
Figure 5. Denoising and result of the first dataset: (a) Gray-scale image rendered using intensity values; (b) A horizontal profile of the DSM (red crossed symbols indicate outliers); and (c) The corresponding result after denoising.

\section{CONCLUSION}

Denoising is a crucial pre-processing step for airborne LiDAR point cloud. In this study, a novel denoising algorithm based on EMD for airborne LiDAR point cloud was the first time developed. The proposed algorithm first decomposed point cloud into a series of IMFs. By innovatively applying OTSU algorithm to these IMFs, the noise-dominated components can be determined and then were filtered according to soft thresholding method. Finally, noise points were detected automatically by comparing observed elevations and reconstructed elevations.

To verify the validity and robustness of the proposed method, three datasets located at three different cities in China were tested. The experiments demonstrate that the proposed method exhibits a good performance in denoising both high and low outliers at the areas with various terrain features (e.g., residential buildings, roads, forests, farmlands, rivers, etc.), thereby providing a good foundation for the post-processing of airborne LiDAR point cloud, such as filtering, building reconstruction, road extraction, and so on.

\section{ACKNOWLEDGEMENTS}

The authors would like to thank the Fund from National Science Foundation (NSF) (41801325, 41861052, 41874001), National Key Research and Development Program of China (2017YFB0503704), Education Department of Jiangxi Province (GJJ170449), Key Laboratory for Digital Land and Resources of Jiangxi Province, East China University of Technology (DLLJ201806), East China University of Technology Ph. D. Project (DHBK2017155) for their financial support.

\section{REFERENCES}

Brovelli, M.A., Cannata, M., Longoni, U.M., 2002. Managing and processing LiDAR data within GRASS. In: Proceedings of the Open Source GIS-GRASS Users Conference, Trento, Italy, 11-13 September.pp. 1-29.

Chen, Q., Gong, P., Baldocchi, D. and Xie, G., 2007. Filtering Airborne Laser Scanning Data with Morphological Methods. 
Photogrammetric Engineering \& Remote Sensing, 73(2): pp. 175-185.

Haugerud, R.A. and Harding, D.J., 2001. Some algorithms for virtual deforestation (VDF) of LiDAR topographic survey data. International Archives of Photogrammetry \& Remote Sensing, pt. 3/W4, vol. 34, 211-218.

Hui, Z., Hu, Y., Yevenyo, Y.Z. and Yu, X., 2016. An Improved Morphological Algorithm for Filtering Airborne LiDAR Point Cloud Based on Multi-Level Kriging Interpolation. Remote Sensing, 8(1): 35.

Li, Y. et al., 2013. A gradient-constrained morphological filtering algorithm for airborne LiDAR. Optics \& Laser Technology, 54: 288-296.

Li, Y., Yong, B., Wu, H., An, R. and Xu, H., 2014. An Improved Top-Hat Filter with Sloped Brim for Extracting Ground Points from Airborne Lidar Point Clouds. Remote Sensing, 6(12): 12885-12908.

Lin, X. and Zhang, J., 2014. Segmentation-Based Filtering of Airborne LiDAR Point Clouds by Progressive Densification of Terrain Segments. Remote Sensing, 6(2): 1294-1326.

Mongus, D. and Žalik, B., 2012. Parameter-free ground filtering of LiDAR data for automatic DTM generation. ISPRS Journal of Photogrammetry and Remote Sensing, 67: 1-12.

Mongus, D., Lukač, N. and Žalik, B., 2014. Ground and building extraction from LiDAR data based on differential morphological profiles and locally fitted surfaces. ISPRS Journal of Photogrammetry and Remote Sensing, 93: 145-156.

Otsu, N., 1979. A Threshold Selection Method from Gray-Level Histograms. IEEE Transactions on Systems Man \& Cybernetics, 9(1): 62-66.

Silván-Cárdenas, J.L. and Wang, L., 2006. A multi-resolution approach for filtering LiDAR altimetry data. ISPRS Journal of Photogrammetry and Remote Sensing, 61(1): 11-22

Sithole, G. and Vosselman, G., 2004. Experimental comparison of filter algorithms for bare-Earth extraction from airborne laser scanning point clouds. ISPRS Journal of Photogrammetry and Remote Sensing, 59(1-2):85-101.

Wang, W., Wang, J. and Sun, G., 2009. Noise reduction and modeling methods of TLS point cloud based on R-tree, Urban Remote Sensing Event, pp. 1-5.

Yang, B., Huang, R., Dong, Z., Zang, Y. and Li, J., 2016. Twostep adaptive extraction method for ground points and breaklines from lidar point clouds. ISPRS Journal of Photogrammetry and Remote Sensing, 119: 373-389. 